\title{
A novel prognostic nomogram for patients with surgically resected perihilar cholangiocarcinoma: a SEER-based study
}

\author{
Pengfei Li, Lujun Song \\ Department of General Surgery, Zhongshan Hospital, Fudan University, Shanghai, China \\ Contributions: (I) Conception and design: P Li; (II) Administrative support: L Song; (III) Provision of study materials or patients: P Li; (IV) Collection \\ and assembly of data: P Li; (V) Data analysis and interpretation: All authors; (VI) Manuscript writing: All authors; (VII) Final approval of manuscript: \\ All authors. \\ Correspondence to: Lujun Song. Department of General Surgery, Zhongshan Hospital, Fudan University, Shanghai 200032, China. \\ Email: song.lujun@zs-hospital.sh.cn.
}

Background: This study aimed to compare the predictive efficacy of four different lymph node (LN) staging systems on the overall survival (OS) of patients with surgically resected perihilar cholangiocarcinoma (pCCA), and construct a novel prognostic nomogram to predict OS in pCCA patients.

Methods: Patients with pCCA that underwent surgical resection between 2004 to 2016 were selected from the Surveillance, Epidemiology, and End Results (SEER) database (n=1,173). Patients were randomly divided into a modeling cohort and an internal verification cohort. To compare the prognostic efficacy of four different $\mathrm{N}$ staging systems [American Joint Committee on Cancer (AJCC) $7^{\text {th }}$ and $8^{\text {th }}$ edition $\mathrm{N}$ stages, lymph node ratio (LNR), and log odds of positive lymph nodes (LODDS)], we used three different evaluation methods: Harrell's index of concordance (C-index), Akaike information criterion (AIC), and area under the receiver operating characteristic (ROC) curve (AUC). Multivariate analysis was used to identify independent prognostic factors and validate LODDS in the modeling cohort. A nomogram was then constructed to predict 1-, 3-, and 5-year survival. The nomogram was validated using Harrell's C-indexes and calibration curves.

Results: Of the four different $\mathrm{N}$ staging methods, LODDS was considered to be the most effective LN staging system for evaluating the prognosis of patients with surgically resected pCCA, according to the values calculated for C-index, AUC, and AIC. After validation by C-indexes and calibration curves, the constructed nomogram accurately predicted the OS of pCCA patients.

Conclusions: For patients with surgically resected pCCA, LODDS was found to be the most accurate N staging system. The novel LODDS-based nomogram constructed in this study provides an accurate method for predicting patient survival in pCCA.

Keywords: Perihilar cholangiocarcinoma (pCCA); log odds of positive lymph nodes (LODDS); nomogram

Submitted Apr 04, 2020. Accepted for publication Sep 24, 2020.

doi: $10.21037 / \mathrm{atm}-20-3130$

View this article at: http://dx.doi.org/10.21037/atm-20-3130

\section{Introduction}

Cholangiocarcinoma (CCA) is a hepatobiliary carcinoma with features of cholangiocyte differentiation, and is the most common malignancy of biliary origin (1-3). Clinically, CCA can be divided into intrahepatic cholangiocarcinoma (iCCA), perihilar cholangiocarcinoma (pCCA), and distal cholangiocarcinoma (dCCA) subtypes, according to tumor location $(4,5)$. These three subtypes of CCA differ not only in their anatomical locations, but also in their pathology, epidemiology, and treatment options $(3,6)$. The largest subtype of CCA is pCCA, accounting for more than $50 \%$ of the incidence of CCA (7). For pCCA that is detected early, 
thorough surgical resection is the most effective method to improve patient survival $(6,8)$, along with lymph node (LN) dissection (6). Therefore, establishing a link between the degree of LN dissection and the overall survival (OS) of patients with pCCA is particularly important for accurately predicting prognosis.

Currently, the most commonly used LN staging system is the $\mathrm{N}$ stage system proposed by the American Joint Committee on Cancer (AJCC) $(9,10)$. In the $7^{\text {th }}$ edition of the AJCC staging system, the definition of LN staging was based on the location of the LNs to be dissected, where LN metastasis around the tumor was classified as $\mathrm{N} 1$, and further LN metastasis was classified as N2 (9). The $8^{\text {th }}$ edition of the AJCC LN staging system is based on the number of positive lymph nodes (PLNs) (10). Although the AJCC has proposed this new LN staging system, its clinical effectiveness remains to be determined. The degree of actual LN dissection during surgery may affect the number of PLNs detected $(11,12)$. To address the impact of the number of $\mathrm{LN}$ dissections on the detection of PLNs, experts have proposed new methods for improving the staging system of LN metastasis. For example, lymph node ratio (LNR) is considered to be a prognostic LN staging criterion for many types of tumor, including colorectal cancer and thyroid cancer (13-15). Log odds of positive lymph nodes (LODDS) was first shown to be associated with the prognosis of stage III colon cancer in 2008, has subsequently proven to be a good predictor of patient prognosis in a variety of tumors, and therefore, is considered to be an effective LN staging system (15-17).

A nomogram is an emerging tool that uses independent prognostic factors acquired from multivariate regression analysis to predict the OS of different cancer types $(11,18-20)$. In the present study, univariate cox regression analysis showed that four different $\mathrm{N}$ staging systems (AJCC $7^{\text {th }}$ and $8^{\text {th }}$ edition $\mathrm{N}$ stages, LODDS, and LNR) were independent prognostic factors for pCCA. We then evaluated whether LODDS was the most effective system for LN staging by using three different models: Harrell's index of concordance (C-index), the Akaike information criterion (AIC), and the area under the receiver operating characteristic (ROC) curve (AUC). Based on LODDS and other independent prognostic factors, we established a novel nomogram for predicting OS in patients with surgically resected pCCA. We present the study in accordance with the STROBE reporting checklist (available at http://dx.doi. org/10.21037/atm-20-3130).

\section{Methods}

\section{Patient selection}

We collected the clinical data of patients with pCCA between 2004 to 2016 from the SEER database (http:// seer.cancer.gov/). This database covers approximately $28 \%$ of the US population and $97 \%$ of tumor categories. The exclusion criteria for patient data were as follows: patients without a pathological diagnosis; patients who did not undergo surgery or patients without surgery information; patients with uncertain $\mathrm{LN}$ resection or missing information concerning regional node examination; patients with unclear data about sex, race, pathological grade or tumor size; patients without specific tumor-node-metastasis (TNM staging). Because information related to the AJCC $7^{\text {th }}$ edition $\mathrm{N}$ staging system could not be obtained from 2004 to 2009, we used the collaborative staging (CS) LN system [2004-2015] from the SEER database to transfer the $\mathrm{N}$ stage into the $7^{\text {th }} \mathrm{N}$ stage, and then excluded patients without specific TNM staging. Our data selection method is shown below in Figure 1. The selected patients were randomly divided into a validation cohort and a modeling cohort by a ratio of approximately 1 to 3 , similar to previous studies $(18,21)$. The study was conducted in accordance with the Declaration of Helsinki (as revised in 2013) (22). This project received an ethical exemption from the Ethics Committee of Zhongshan Hospital Affiliated to Fudan University. The SEER database is an open access database, and all patient information has been de-identified, so informed consent was waived.

Several variables were examined, including sex, race, age of diagnosis, tumor size, AJCC TNM stage, histology, pathological grade, radiation, and chemotherapy. The AJCC TNM staging system was used to identify the stage of pCCA. Although the AJCC T stage did not change from the $7^{\text {th }}$ to the $8^{\text {th }}$ edition, it differed from the $6^{\text {th }}$ edition AJCC $\mathrm{T}$ stage, and could therefore only be transferred from the $7^{\text {th }}$ edition to the $6^{\text {th }}$ edition. Because a portion of the data were collected prior to 2010, we used the AJCC $6^{\text {th }}$ edition T staging system instead of the $7^{\text {th }}$ edition to establish our prognostic model. $M$ stage has remained unchanged since the AJCC $6^{\text {th }}$ edition, hence we used the $8^{\text {th }}$ edition. The AJCC $8^{\text {th }}$ edition $\mathrm{N}$ stage values were transformed into the number of PLNs: 0 for N0, $1-3$ for N1, and 4 or more for N2. The variables CS tumor size and tumor size (2016+) were used to extract tumor size. The best cutoff for tumor size was calculated using $\mathrm{X}$-tile software, and tumor size was grouped as $<1.7,1.7-3.3$, and $\geq 3.4 \mathrm{~cm}$ (Figure S1A,B,C) (23). 


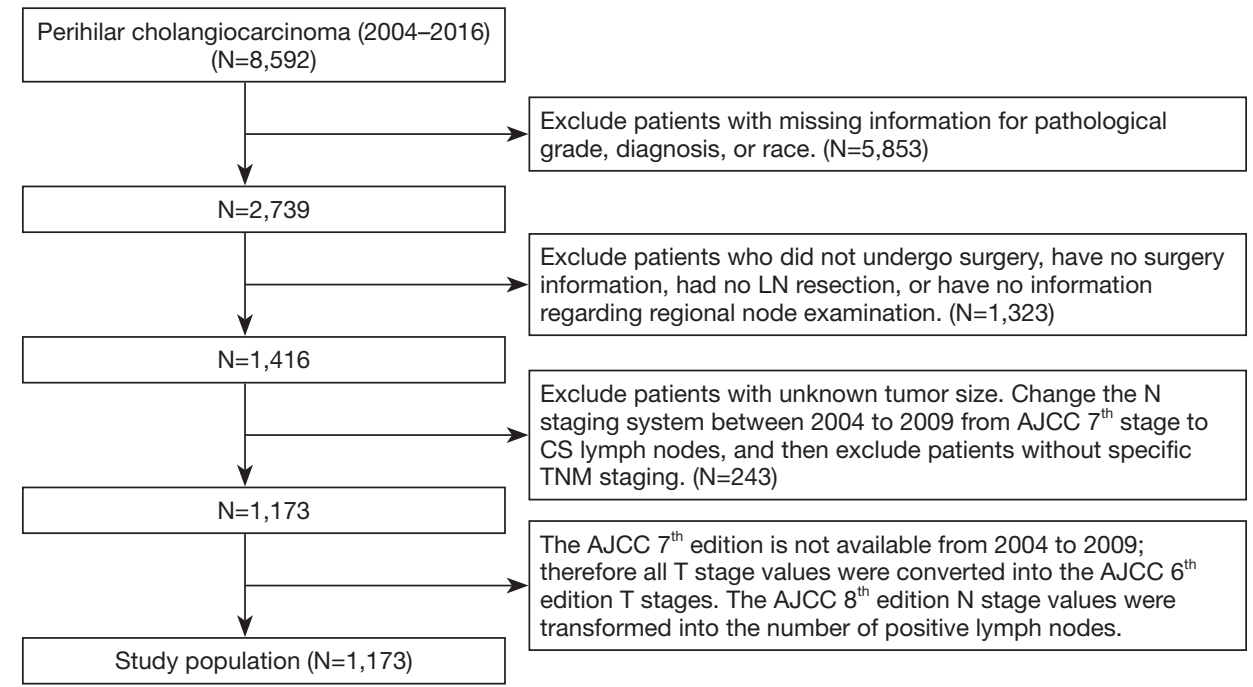

Figure 1 Flow diagram of the study selection process. AJCC, American Joint Committee on Cancer; CS, collaborative staging; TNM, tumor-node-metastasis.

\section{LODDS and LNR systems}

LODDS was calculated by using the following formula: $\log [(0.05+$ amount of PLNs $) /(0.05+$ amount of negative LNs)]. LODDS in our cohort ranged from -3.11 to 2.45. Meanwhile, LNR was calculated by using the following formula: amount of PLNs/amount of resected LNs. We used the $\mathrm{X}$-tile software to derive the best cutoff for LNR and LODDS (Figure S1D,E,F,G,H,I) (24). LODDS was divided into LODDS1 (-3.11 to -1.32$)$, LODDS2 (-1.32 to -0.39 ), and LODDS3 ( -0.39 to 2.45 ). LNR was divided into LNR1 (0-0.06), LNR2 (0.06-0.27), and LNR3 (0.27-1).

\section{Statistical analysis}

Statistical analyses were carried out using $\mathrm{R}$ software version 3.6.1 (The R Foundation for Statistical Computing, Vienna, Austria) and SPSS 20.0 (SPSS Inc., Chicago, IL, USA). Pearson's Chi-squared test was used to compare clinical characteristics. The univariate and multivariate analyses were conducted using Cox proportional hazards regression model, and hazard ratio (HR) and $95 \%$ confidence interval (CI) were obtained. Statistically significant variables $(\mathrm{P}<0.05)$ from the univariate analysis were brought into the multivariate analysis. The C-index, AIC, and AUC values were used to compare the prognostic ability of the AJCC $7^{\text {th }}$ and $8^{\text {th }}$ edition N stages, LODDS, and LNR for pCCA. The Kaplan-Meier method was used to estimate OS according to the four $\mathrm{N}$ stages, and survival curves were analyzed using the log-rank test. $\mathrm{P}$ values were calculated using two-tailed tests, and a $\mathrm{P}$ value $<0.05$ was considered statistically significant.

The rms package within $\mathrm{R}$ was used to formulate the nomogram according to LODDS and other independent prognostic factors from the multivariate analysis. Consistent with previous studies, C-index, ROC curves, and calibration curves were used to evaluate the nomogram (18).

\section{Result}

\section{Clinicopathological characteristics of patients}

In total, 1,173 eligible patients with surgically resected pCCA from 2004 to 2016 were included in the study as the initial cohort. From the initial cohort, patients were then separated into a modeling cohort and an internal verification cohort, with 881 and 292 patients respectively. The clinical and pathological characteristics of patients are listed in Table 1. No significant differences were found between the two cohorts in regards to clinical characteristics and treatment methods. Patients over 65 years old were considered elderly, with 716 patients older than 65 years old $(61.0 \%)$ and 457 patients younger than 65 years old (39.0\%). Overall, $717(61.1 \%)$ patients were male, and the most common race was white $(896,76.4 \%)$. The most common tumor grade was moderately differentiated (617, 52.6\%), followed by poorly differentiated (380, 32.4\%). According to the AJCC TNM cancer staging system, the majority of 
Page 4 of 13

Li and Song. Nomogram for patients with surgically resected pCCA

Table 1 Clinical and pathological features of patients with surgically resected pCCA

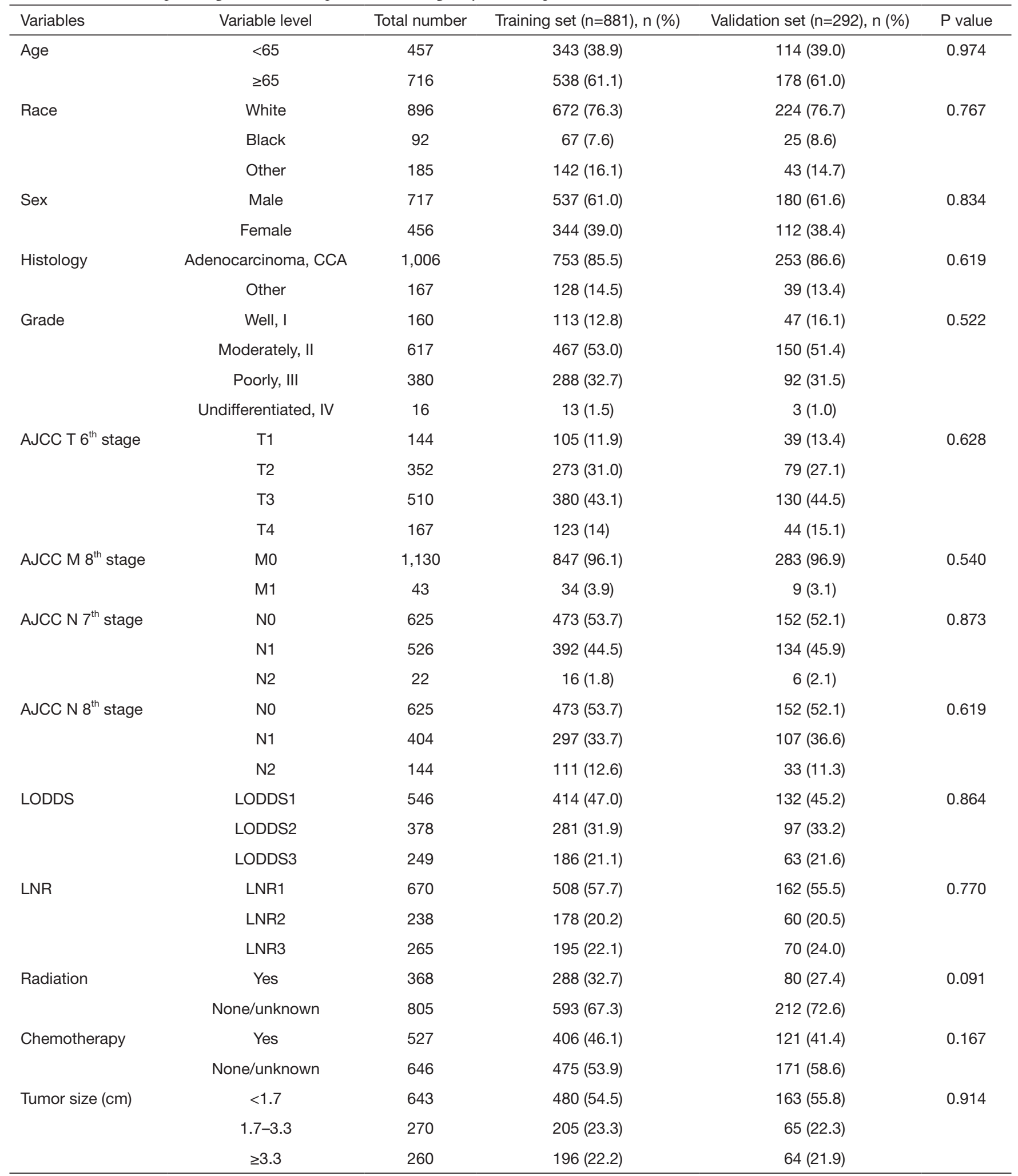

pCCA, perihilar cholangiocarcinoma; CCA, cholangiocarcinoma; AJCC, American Joint Committee on Cancer; TNM, tumor-nodemetastasis; LODDS, log odds of positive lymph nodes; LNR, lymph node ratio. 
patients were classified as T3 $(510,43.5 \%)$ and M0 (1,130, $96.3 \%)$. For tumor size, $643(54.8 \%)$ patients had a tumor size smaller than $1.7 \mathrm{~cm}$, and $270(23.0 \%)$ patients and 260 (22.2\%) patients had a tumor size of $1.7-2.3$ and $\geq 3.3 \mathrm{~cm}$, respectively.

\section{Comparing different $N$ stages in the prognosis of pCCA patients after surgical resection}

In total, the median follow-up time was 19 (range: 0-155) months and the median OS was 23 months (95\% CI: 20.98-25.02). In the training set, the univariate analysis showed that age, grade, histology, tumor size, $T$ stage, $M$ stage, radiation, chemotherapy, AJCC $7^{\text {th }}$ and $8^{\text {th }}$ edition $\mathrm{N}$ stage, LODDS, and LNR were significantly correlated with OS $(\mathrm{P}<0.05)$ (Table 2$)$. Among the 1,173 patients, $548(46.7 \%)$ patients had LN metastasis. According to the $7^{\text {th }}$ and $8^{\text {th }}$ edition AJCC N staging system, $526(44.8 \%)$ patients and $404(34.4 \%)$ patients were N1, respectively, while $22(1.9 \%)$ patients and $144(12.3 \%)$ patients were N2, respectively; LNR and LODDS accounted for 546 (46.5\%), 378 (32.2\%), 249 (21.2\%), 670 (57.1\%), 238 (20.3\%), and $265(22.6 \%)$ patients from level 1 to 3 , respectively. Through Kaplan-Meier survival analysis, we concluded that the four different LN metastasis stages were statistically significant for $\mathrm{OS}$ in their respective strata (Figure 2). The C-indexes for the AJCC $7^{\text {th }}$ and $8^{\text {th }}$ edition $\mathrm{N}$ stages, and LODDS and LNR were 0.598, $0.599,0.616$, and 0.608 , respectively. The AIC values of the four $\mathrm{N}$ stages were 7394, 7381, 7375, and 7381, respectively. The AUC values of the ROC curve of the four $\mathrm{N}$ stages for 3 -year OS were $0.661,0.674,0.691$, and 0.671 , respectively. The AUC values of the ROC curve of the four $\mathrm{N}$ stages for 5-year OS were 0.651, 0.660, 0.675, and 0.655 , respectively (Table 3). LODDS was found to be the optimal staging system in all three evaluation modes, and was therefore considered to be the best LN staging system for evaluating prognosis in pCCA patients.

Table 2 Univariate cox regression analysis of OS for patients with surgically resected pCCA in the training cohort

\begin{tabular}{|c|c|c|c|}
\hline Characteristics & \multicolumn{3}{|c|}{ Univariate analysis } \\
\hline Age (year) & & & $<0.001^{\star \star *}$ \\
\hline$<65$ & Reference & & \\
\hline$\geq 65$ & 1.365 & $1.157-1.610$ & $<0.001^{\star \star \star}$ \\
\hline White & Reference & & \\
\hline Black & & & 0.564 \\
\hline Other & & & 0.386 \\
\hline Sex & & & 0.502 \\
\hline Histology & & & $0.014^{*}$ \\
\hline Adenocarcinoma and CCA & Reference & & \\
\hline Other & 0.744 & $0.587-0.942$ & $0.014^{*}$ \\
\hline Grade & & & $0.011^{*}$ \\
\hline 1 & Reference & & \\
\hline II & 1.024 & $0.794-1.321$ & 0.854 \\
\hline III & 1.352 & $1.037-1.762$ & $0.026^{*}$ \\
\hline IV & 0.934 & $0.43-2.029$ & 0.863 \\
\hline
\end{tabular}

Table 2 (continued) 
Table 2 (continued)

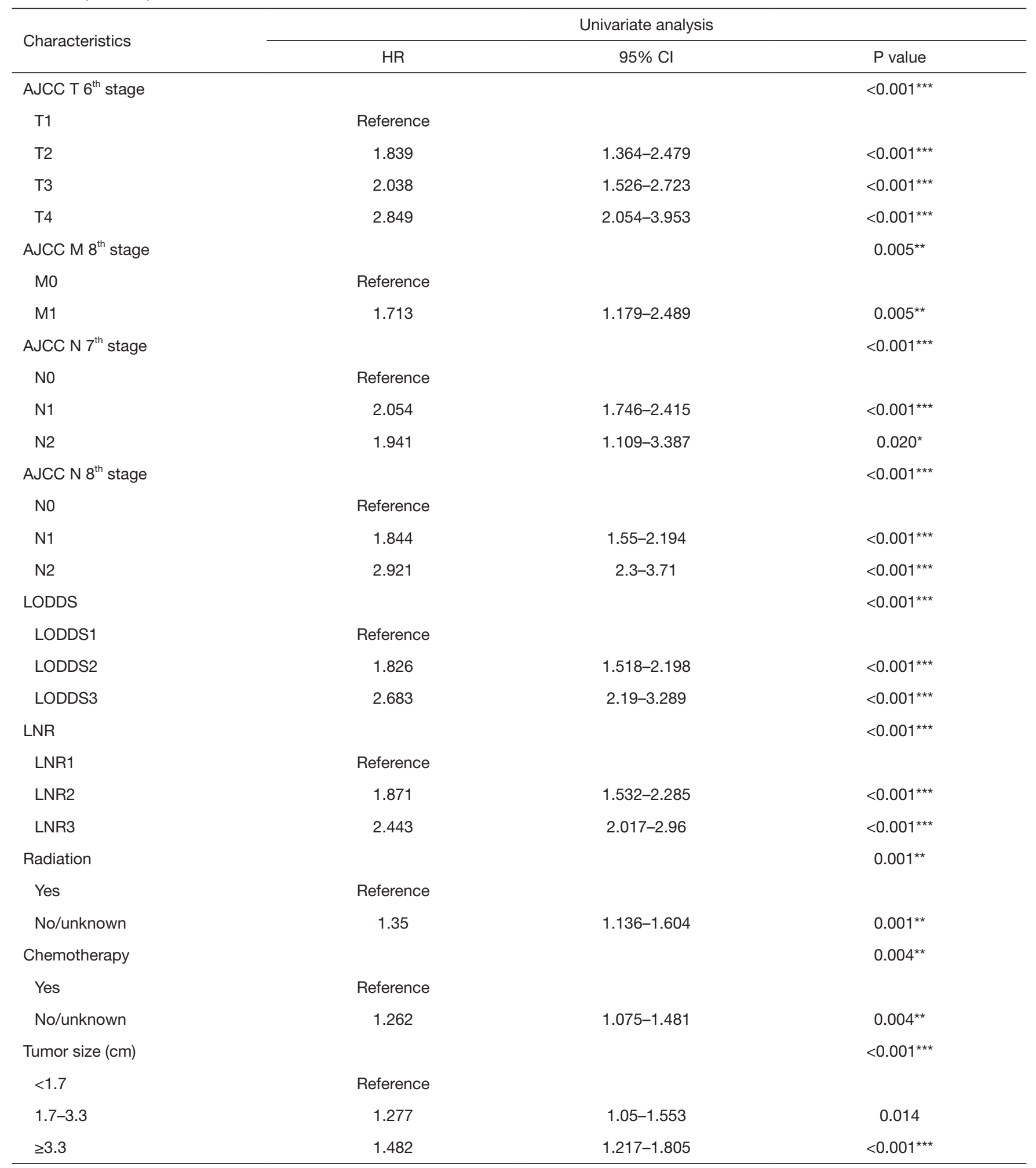

*, $\mathrm{P}<0.05$; ${ }^{* *}, \mathrm{P}<0.01$; ${ }^{* \star *}, \mathrm{P}<0.001$. OS, overall survival; $\mathrm{pCCA}$, perihilar cholangiocarcinoma; HR, hazard ratio; Cl, confidence interval; CCA, cholangiocarcinoma; AJCC, American Joint Committee on Cancer; TNM, tumor-node-metastasis; LODDS, log odds of positive lymph nodes; LNR, lymph node ratio. 

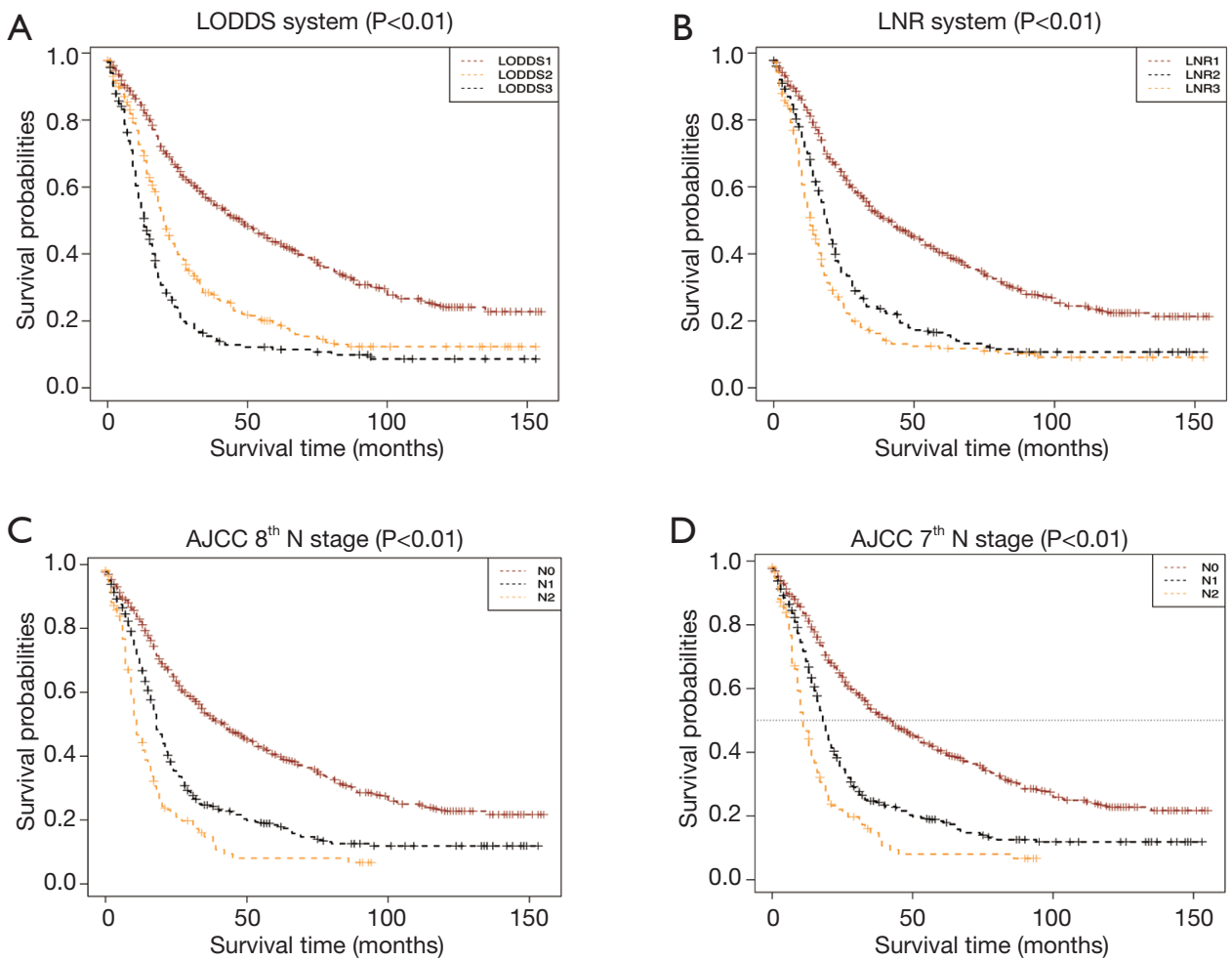

Figure 2 Kaplan-Meier curves stratified by four different N staging systems. (A) LODDS system, (B) LNR system, (C) AJCC $8^{\text {th }}$ N stage, (D) AJCC $7^{\text {th }} \mathrm{N}$ stage. LODDS, log odds of positive lymph nodes; LNR, lymph node ratio; AJCC, American Joint Committee on Cancer; OS, overall survival.

Table 3 Comparison of the prognostic efficacy of four LN staging systems

\begin{tabular}{lcccc}
\hline \multirow{2}{*}{ System } & C-index & AIC & \multicolumn{2}{c}{ AUC } \\
\cline { 3 - 4 } & & & 3-year survival & 5-year survival \\
\hline LODDS & 0.616 & 7,375 & 0.691 & 0.675 \\
LNR & 0.608 & 7,381 & 0.671 & 0.655 \\
AJCC $7^{\text {th }} \mathrm{N}$ stage & 0.598 & 7,394 & 0.661 & 0.651 \\
AJCC $8^{\text {th }} \mathrm{N}$ stage & 0.599 & 7,381 & 0.674 & 0.660 \\
\hline
\end{tabular}

LN, lymph node; AIC, Akaike information criterion; AUC, area under the ROC curve; LODDS, log odds of positive lymph nodes; LNR, lymph node ratio; AJCC, American Joint Committee on Cancer.

\section{Constructing prognostic nomograms for OS}

The prognostic nomogram was constructed using LODDS and other independent survival predictors obtained from the results of the multivariate analysis (Table 4, Figure 3). The training cohort was used to construct the nomogram which describes the 1-, 3-, and 5-year OS rates. The C-index for OS prediction was 0.665 (95\% CI: 0.628-0.687). The ROC curves for the nomogram-predicted OS at 1,3 , and
5 years are depicted in Figure 4. AUC values in the training cohort were $0.724,0.719$, and 0.710 , respectively.

The results of internal verification showed that the C-index of the nomogram-predicted survival for surgically resected pCCA was 0.650 (95\% CI: 0.607-0.693). The calibration curves showed that the above prediction model can accurately predict the 1-, 3-, and 5-year survival probability of pCCA in both the training cohort and 
Page 8 of 13

\section{Li and Song. Nomogram for patients with surgically resected pCCA}

Table 4 Multivariate cox regression analysis of OS for patients with surgically resected pCCA in the training cohort

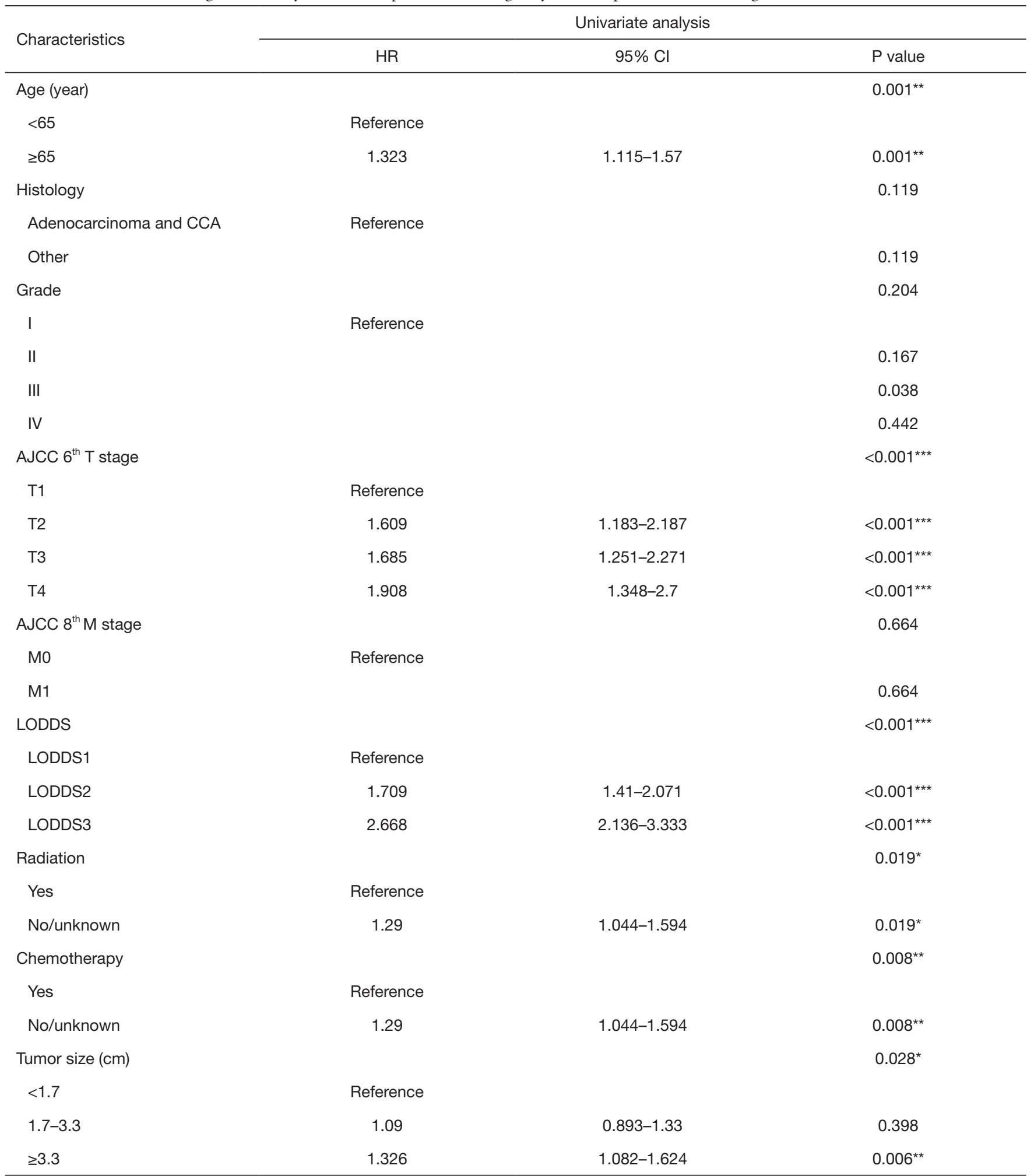

*, $\mathrm{P}<0.05$; ${ }^{\star *}, \mathrm{P}<0.01$; ${ }^{\star \star \star}, \mathrm{P}<0.001$. OS, overall survival; $\mathrm{pCCA}$, perihilar cholangiocarcinoma; HR, hazard ratio; $\mathrm{Cl}$, confidence interval; CCA, cholangiocarcinoma; AJCC, American Joint Committee on Cancer; TNM, tumor-node-metastasis; LODDS, log odds of positive lymph nodes. 


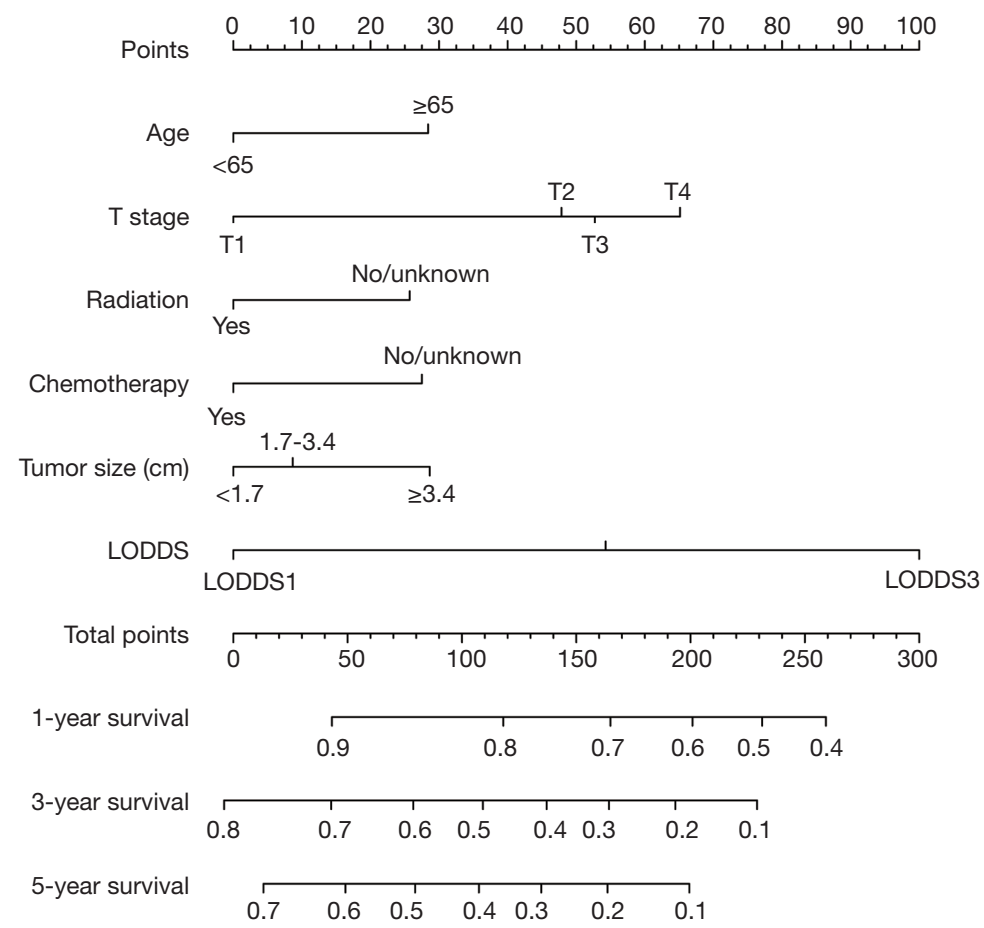

Figure 3 Nomogram for predicting the OS of patients with surgically resected pCCA. OS, overall survival; pCCA, perihilar cholangiocarcinoma; LODDS, log odds of positive lymph nodes.

internal validation cohort (Figure 5). In the validation cohort, the AUC values of the ROC curve for 1-, 3-, and 5 -year OS were $0.693,0.718$, and 0.734 , respectively.

\section{Discussion}

PCCA accounts for the majority of CCA cases, and surgical resection is the most effective method for improving the OS of patients $(7,24)$. Along with the depth of invasion, tumor size, and pathological stage, LN metastasis is considered to be a critical factor affecting the prognosis of cancer patients, including pCCA patients $(7,14,24,25)$. Accurate methods for linking LN dissection and patient prognosis have remained elusive. The LN staging system provided by the AJCC $7^{\text {th }}$ edition $\mathrm{N}$ stage has been widely used to evaluate $\mathrm{LN}$ metastasis and the prognosis of patients (9). In the $7^{\text {th }}$ edition, it is divided into three stages between N0 and N2 according to the presence and location of LN metastasis: $\mathrm{N} 0$ is for no LN metastasis; $\mathrm{N} 1$ is for regional LN metastases (including in the common bile duct, cystic duct, hepatic artery, and portal vein); and N2 is for metastasis around the abdominal aorta, the vena cava, superior mesenteric artery, and abdominal LNs (9).
With the advance of clinical research, several studies have emerged suggesting that considering the location of metastasis often ignores the impact of the number of LN metastases (NLNM) on the degree of LN metastasis (26). The AJCC $8^{\text {th }}$ edition LN staging system confirmed this view (10). The $8^{\text {th }}$ edition LN staging system is based on the NLNM: N0, non-PLNs; N1, 1 to 3 LNs; N2, 4 or more LNs (10). However, the efficacy of evaluations using the AJCC $8^{\text {th }}$ edition LN staging system remains to be verified. In addition to the AJCC $8^{\text {th }}$ edition staging system, there are two other unique evaluation systems used clinically: LNR and LODDS. LNR is calculated as follows: amount of PLNs/amount of resected LNs $(14,15)$. Meanwhile, LODDS is defined as the $\log$ of the ratio between the number of positive nodes and the number of negative nodes (11). Different from the AJCC $8^{\text {th }}$ edition staging system, LNR and LODDS also consider the effect of the total amount of LNs removed during surgery on the amount of PLNs, standardized and converted to NMLN. LNR has its limitations, as all surgically resected LNs are considered positive. For example, whether there is 1 PLN or more than 10 PLNs, the LNR value is always 1. Many studies have also questioned the application of LNR 

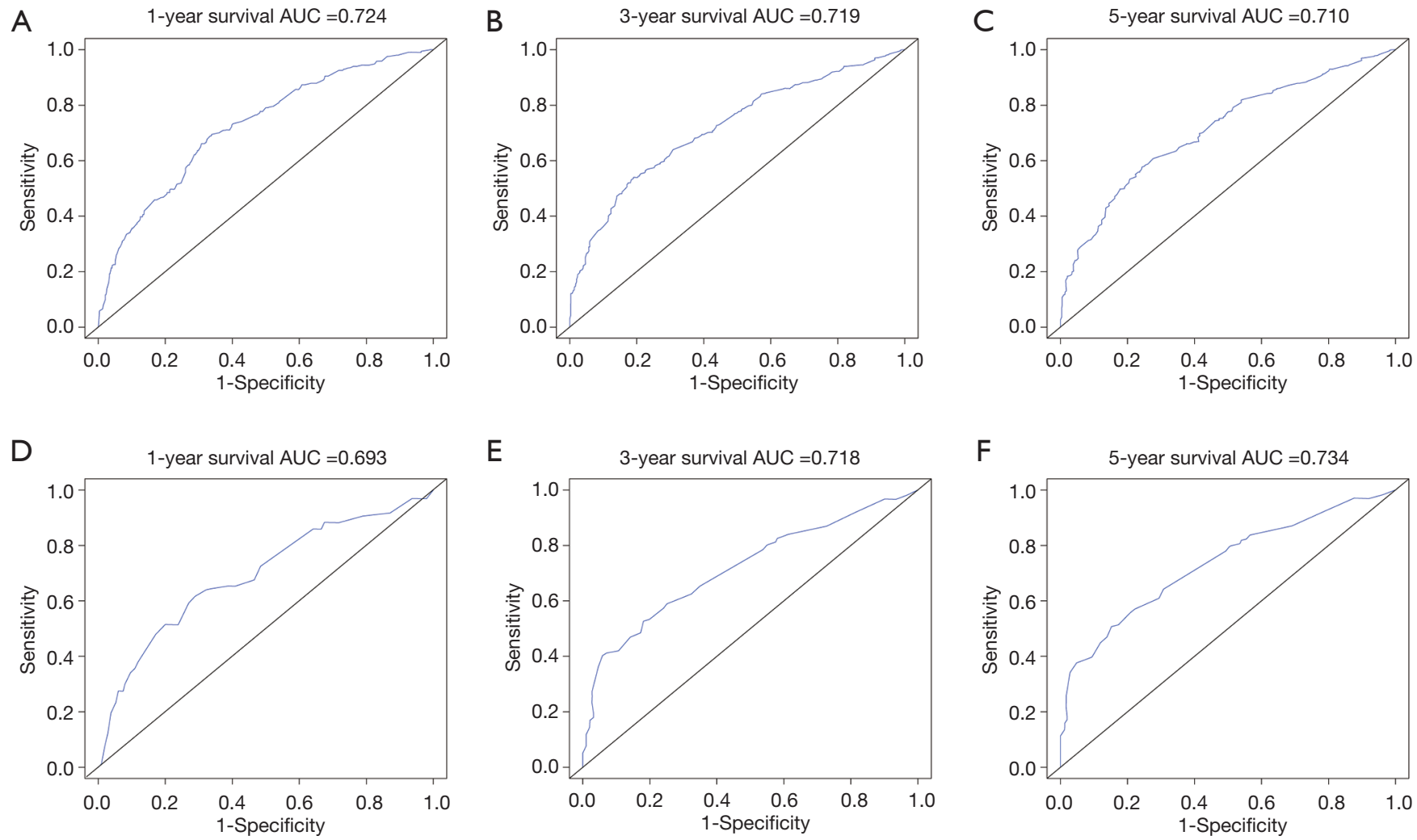

Figure 4 AUC values and ROC curves of the nomogram for the predicted 1-, 3-, and 5-year OS rates for the training cohort (A,B,C) and the internal validation cohort $(\mathrm{D}, \mathrm{E}, \mathrm{F})$. The $\mathrm{X}$-axis represents the nomogram-predicted OS probability. AUC, area under the curve; ROC, receiver operating characteristic; OS, overall survival.

when all resections are positive, which makes it difficult to describe the LN metastasis in this situation $(11,12)$. These limitations contributed to the creation of the LODDS evaluation system. In evaluating LN metastasis, LODDS uses a base number and negative LNs as the denominator, and thus there is a 10-fold difference between 1 positive and 10 PLNs before log changes are made. This means that the LODDS system can be used in situations that cannot be determined by the LNR method. As we can see, different $\mathrm{LN}$ staging systems have advantages and disadvantages, and some controversy remains over which $\mathrm{LN}$ staging system is the most effective $(12,15,26,27)$.

In the present study, we developed a novel method to evaluate the efficacy of four LN staging systems that predict the OS of patients with surgically resected pCCA: the AJCC $7^{\text {th }}$ and $8^{\text {th }}$ edition $\mathrm{N}$ staging, LODDS, and LNR. The cutoff values for LODDS and LNR were obtained by automatic fitting using X-tile software (23). This software, developed by Yale University, can provide accurate cutoffs for index stratification, and has been applied in the index stratification for evaluation systems of different diseases $(18,28,29)$. First, through using KaplanMeier survival analysis and univariate cox regression analysis, we demonstrated that all four LN staging systems were correlated with OS in patients with surgically resected pCCA $(\mathrm{P}<0.05)$. After calculating $\mathrm{C}$-indexes, AIC values and AUC values, we found LODDS to have the best prognostic evaluation results of the different evaluation systems. This is consistent with the results of previous studies $(11,27)$. For example, Xiao et al. (11) used SEER data from 1,321 patients with surgically resected gallbladder cancer to determine that LODDS had better OS predictive accuracy than other LN staging systems, like LNR. Furthermore, using data from 118 patients with pCCA, Conci et al. (27) demonstrated that LODDS was the most effective staging system compared to other LN staging systems. Along with other independent prognostic factors, we then established a nomogram based on LODDS for the prediction of prognosis in patients with surgically resected pCCA. We then used the 
A

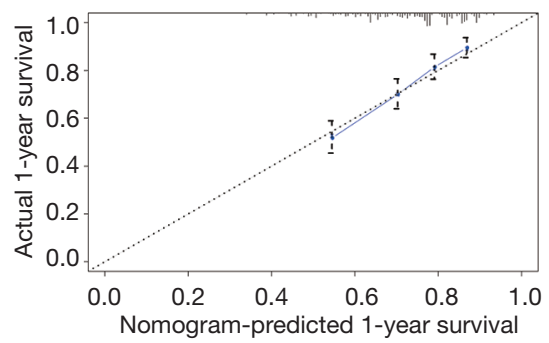

$\mathrm{D}$

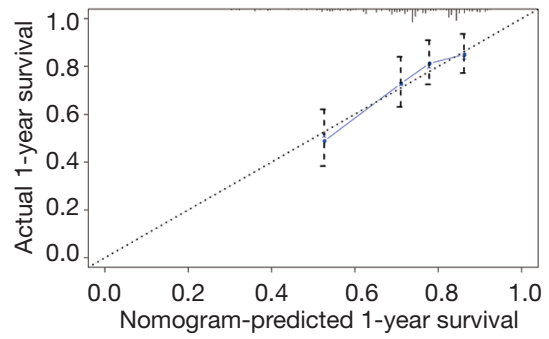

B

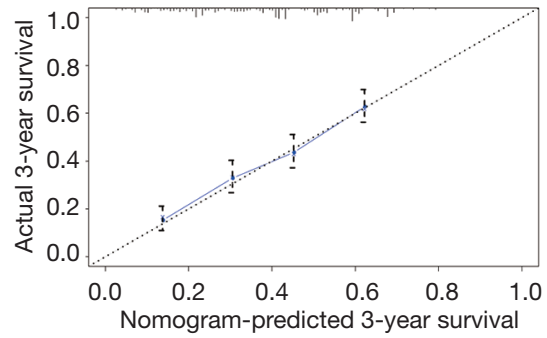

E

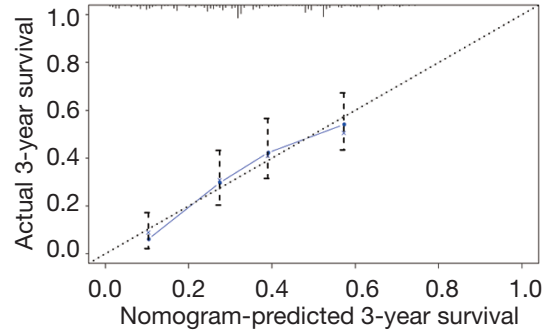

B

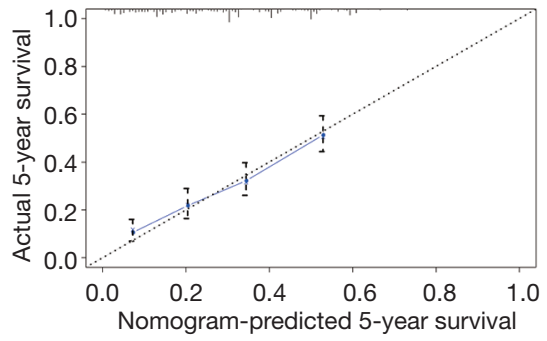

F

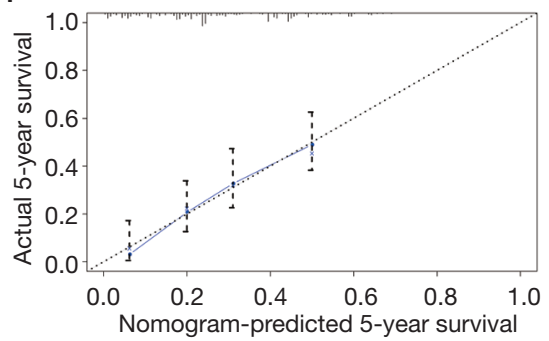

Figure 5 Calibration graphs of the nomogram for the predicted 1-, 3-, and 5-year OS rates for the training cohort (A,B,C) and the internal validation cohort (D,E,F). The X-axis represents the nomogram-predicted OS probability. The Y-axis represents the actually observed OS probability. Diagonal lines represent the ideal nomogram reference. Striped dots represent the nomogram-predicted probability with the $95 \%$ CI. OS, overall survival; CI, confidence interval.

C-index, calibration curves, and ROC curves of an internal verification cohort to confirm that our nomogram could accurately evaluate the prognosis of patients with pCCA. Therefore, to the best of our knowledge, our study is the first population-based, SEER-based study that compares different LN staging systems for patients with surgically resected pCCA.

We acknowledge several limitations in this study. Firstly, this study used retrospective data from the SEER database, which might have lead to selection bias. Secondly, although we used a large sample of data from the SEER database for comparison and modeling, we utilized an internal verification cohort to develop the nomogram. Validation using other external data should be performed to provide further evidence of predictive accuracy. Thirdly, we were unable to obtain data concerning the AJCC $7^{\text {th }}$ edition T stage from the SEER database between 2004 to 2009. Evaluation of the infiltration depth in our nomogram was based on the AJCC $6^{\text {th }}$ edition, which might have reduced the model's predictive accuracy. In future studies, further verification of the effectiveness of LODDS in clinical prognostic evaluations is needed through prospective studies, along with use of more detailed pathological information.
In conclusion, for patients with surgically resected pCCA, LODDS was determined to be the most effective LN staging system. Our nomogram, based on LODDS, is an accurate and convenient method for the assessment of prognosis in patients with pCCA.

\section{Acknowledgments}

We would like to express our gratitude to the SEER program for providing free access to the database.

Funding: This study was supported by grants from the Research Project of Zhongshan Hospital, Fudan University (2016ZSFZ43).

\section{Footnote}

Reporting Checklist: The authors have completed the STROBE reporting checklist. Available at http://dx.doi. org/10.21037/atm-20-3130

Conflicts of Interest: Both authors have completed the ICMJE uniform disclosure form (available at http://dx.doi. org/10.21037/atm-20-3130). The authors have no conflicts of interest to declare. 


\section{Page 12 of 13}

Ethical Statement: The authors are accountable for all aspects of the work in ensuring that questions related to the accuracy or integrity of any part of the work are appropriately investigated and resolved. The study was conducted in accordance with the Declaration of Helsinki (as revised in 2013). This project received an ethical exemption from the Ethics Committee of Zhongshan Hospital Affiliated to Fudan University. The SEER database is an open access database, and all patient information has been de-identified, so informed consent was waived.

Open Access Statement: This is an Open Access article distributed in accordance with the Creative Commons Attribution-NonCommercial-NoDerivs 4.0 International License (CC BY-NC-ND 4.0), which permits the noncommercial replication and distribution of the article with the strict proviso that no changes or edits are made and the original work is properly cited (including links to both the formal publication through the relevant DOI and the license). See: https://creativecommons.org/licenses/by-nc-nd/4.0/.

\section{References}

1. Rizvi S, Gores GJ. Pathogenesis, diagnosis, and management of cholangiocarcinoma. Gastroenterology 2013;145:1215-29.

2. Khan SA, Thomas HC, Davidson BR, et al. Cholangiocarcinoma. Lancet 2005;366:1303-14.

3. Lazaridis KN, Gores GJ. Cholangiocarcinoma. Gastroenterology 2005;128:1655-67.

4. Rizvi S, Eaton J, Yang JD, et al. Emerging technologies for the diagnosis of perihilar cholangiocarcinoma. Semin Liver Dis 2018;38:160-9.

5. Blechacz B, Komuta M, Roskams T, et al. Clinical diagnosis and staging of cholangiocarcinoma. Nat Rev Gastroenterol Hepatol 2011;8:512-22.

6. Rizvi S, Khan SA, Hallemeier CL, et al. Cholangiocarcinoma - evolving concepts and therapeutic strategies. Nat Rev Clin Oncol 2018;15:95-111.

7. DeOliveira ML, Cunningham SC, Cameron JL, et al. Cholangiocarcinoma: thirty-one-year experience with 564 patients at a single institution. Ann Surg 2007;245:755-62.

8. Aloia TA. Precision hilar cholangiocarcinoma surgery. Ann Surg Oncol 2018;25:1103-4.

9. Edge SB, Compton CC. The American Joint Committee on Cancer: the 7th edition of the AJCC cancer staging manual and the future of TNM. Ann Surg Oncol 2010;17:1471-4.

\section{Li and Song. Nomogram for patients with surgically resected pCCA}

10. Chun YS, Pawlik TM, Vauthey JN. 8th edition of the AJCC cancer staging manual: pancreas and hepatobiliary cancers. Ann Surg Oncol 2018;25:845-7.

11. Xiao Z, Shi Z, Hu L, et al. A new nomogram from the SEER database for predicting the prognosis of gallbladder cancer patients after surgery. Ann Transl Med 2019;7:738.

12. Murakami Y, Uemura K, Sudo T, et al. Number of metastatic lymph nodes, but not lymph node ratio, is an independent prognostic factor after resection of pancreatic carcinoma. J Am Coll Surg 2010;211:196-204.

13. Wang W, Shen Z, Shi Y, et al. Accuracy of nodal positivity in inadequate lymphadenectomy in pancreaticoduodenectomy for pancreatic ductal adenocarcinoma: a population study using the US SEER database. Front Oncol 2019;9:1386.

14. Zhang CH, Li YY, Zhang QW, et al. The prognostic impact of the metastatic lymph nodes ratio in colorectal cancer. Front Oncol 2018;8:628.

15. Occhionorelli S, Andreotti D, Vallese P, et al. Evaluation on prognostic efficacy of lymph nodes ratio (LNR) and log odds of positive lymph nodes (LODDS) in complicated colon cancer: the first study in emergency surgery. World J Surg Oncol 2018;16:186.

16. Wang J, Hassett JM, Dayton MT, et al. The prognostic superiority of log odds of positive lymph nodes in stage III colon cancer. J Gastrointest Surg 2008;12:1790-6.

17. Han L, Mo S, Xiang W, et al. Comparison of four lymph node staging systems for predicting prognosis for stage IV rectum cancer. Ann Transl Med 2020;8:111.

18. Li J, Liu L. Overall survival in patients over 40 years old with surgically resected pancreatic carcinoma: a SEERbased nomogram analysis. BMC Cancer 2019;19:726.

19. Yan X, Fu X, Guo ZX, et al. Construction and validation of an eight-gene signature with great prognostic value in bladder cancer. J Cancer 2020;11:1768-79.

20. Li W, Li X, Gao LN, et al. Integrated analysis of the functions and prognostic values of RNA binding proteins in lung squamous cell carcinoma. Front Genet 2020;11:185.

21. Wang Y, Li J, Xia Y, et al. Prognostic nomogram for intrahepatic cholangiocarcinoma after partial hepatectomy. J Clin Oncol 2013;31:1188-95.

22. World Medical Association. World Medical Association Declaration of Helsinki: ethical principles for medical research involving human subjects. JAMA 2013;310:2191-4.

23. Camp RL, Dolled-Filhart M, Rimm DL. X-tile: a new bio-informatics tool for biomarker assessment and 
outcome-based cut-point optimization. Clin Cancer Res 2004;10:7252-9.

24. Groot Koerkamp B, Jarnagin WR. Surgery for perihilar cholangiocarcinoma. Br J Surg 2018;105:771-2.

25. Kitagawa Y, Nagino M, Kamiya J, et al. Lymph node metastasis from hilar cholangiocarcinoma: audit of 110 patients who underwent regional and paraaortic node dissection. Ann Surg 2001;233:385-92.

26. Bagante F, Tran T, Spolverato G, et al. Perihilar cholangiocarcinoma: number of nodes examined and optimal lymph node prognostic scheme. J Am Coll Surg 2016;222:750-9.e2.

27. Conci S, Ruzzenente A, Sandri M, et al. What is the

Cite this article as: Li P, Song L. A novel prognostic nomogram for patients with surgically resected perihilar cholangiocarcinoma: a SEER-based study. Ann Transl Med 2021;9(1):54. doi: 10.21037/atm-20-3130 most accurate lymph node staging method for perihilar cholangiocarcinoma? Comparison of UICC/AJCC $\mathrm{pN}$ stage, number of metastatic lymph nodes, lymph node ratio, and log odds of metastatic lymph nodes. Eur J Surg Oncol 2017;43:743-50.

28. Lin HP, Li SW, Liu Y, et al. Prognostic value of lymph nodes count on survival of patients with distal cholangiocarcinomas. World J Gastroenterol 2018;24:1022-34.

29. Li Y, Liang L, Dai W, et al. Prognostic impact of programed cell death-1 (PD-1) and PD-ligand 1 (PDL1) expression in cancer cells and tumor infiltrating lymphocytes in colorectal cancer. Mol Cancer 2016;15:55. 
A

C

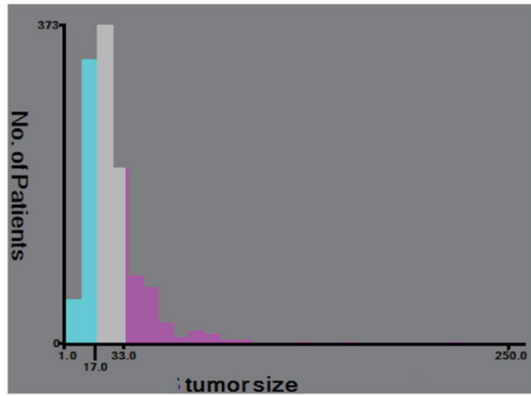

\begin{tabular}{|l|c|c|c|c|c|}
\hline Pt No & $\%$ Total & Events & Rate & Rank & Range \\
\hline \hline 335 & 28.56 & 235 & 70.15 & 0 to 16 & 1.00 thru 17.00 \\
\hline 578 & 49.28 & 401 & 69.38 & 17 to 32 & 18.00 thru 33.00 \\
\hline 260 & 22.17 & 193 & 74.23 & 33 to 76 & 34.00 thru 250.00 \\
\hline 1173 & 100.00 & 829 & 70.67 & 0 to 76 & 1.00 thru 250.00 \\
\hline
\end{tabular}

D

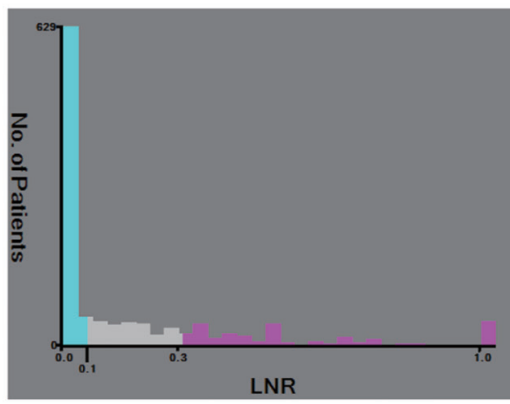

B

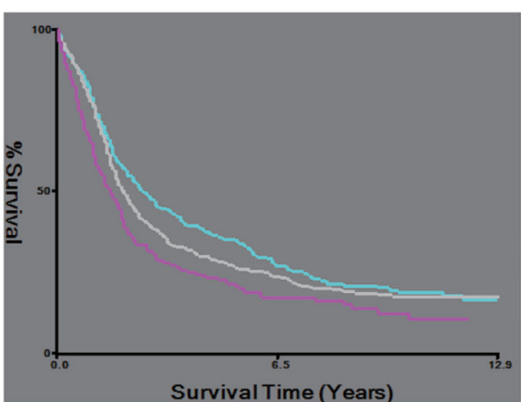

E

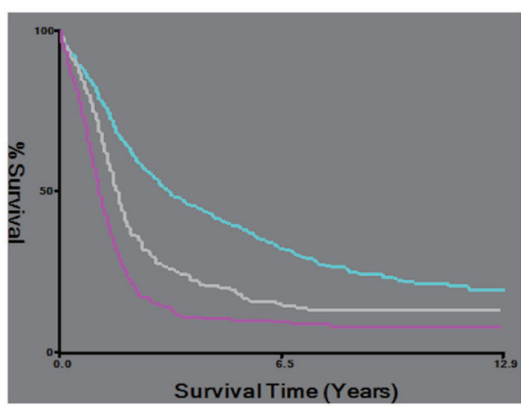

F

\begin{tabular}{|l|c|c|c|c|c|}
\hline Pt No & \% Total & Events & Rate & Rank & Range \\
\hline \hline 658 & 56.10 & 409 & 62.16 & 0 to 17 & 0.00 thru 0.06 \\
\hline 252 & 21.48 & 193 & 76.59 & 18 to 73 & 0.06 thru 0.27 \\
\hline 263 & 22.42 & 227 & 86.31 & 74 to 122 & 0.29 thru 1.00 \\
\hline 1173 & 100.00 & 829 & 70.67 & 0 to 122 & 0.00 thru 1.00 \\
\hline
\end{tabular}

G

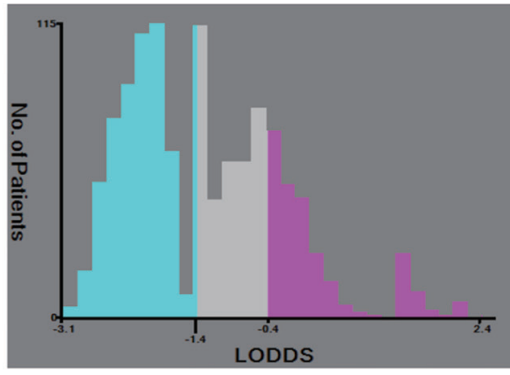

$\mathrm{H}$

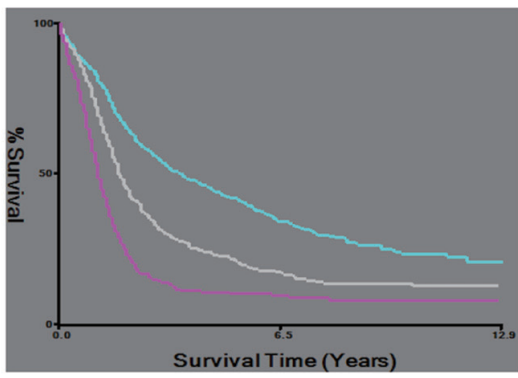

I

\begin{tabular}{|l|c|c|c|c|c|}
\hline Pt No & $\%$ Total & Events & Rate & Rank & Range \\
\hline \hline 547 & 46.63 & 330 & 60.33 & 0 to 48 & -3.11 thru -1.36 \\
\hline 365 & 31.12 & 274 & 75.07 & 49 to 142 & -1.32 thru -0.40 \\
\hline 261 & 22.25 & 225 & 86.21 & 143 to 217 & -0.39 thru 2.45 \\
\hline 1173 & 100.00 & 829 & 70.67 & 0 to 217 & -3.11 thru 2.45 \\
\hline
\end{tabular}

Figure S1 X-tile plots identifying the cut-off of tumor size (A,B,C), LNR (D,E,F), LODDS (G,H,I). LNR, lymph node ratio; LODDS, log odds of positive lymph nodes. 